\title{
Photometric Investigation of Hot Exoplanets: TrES-3b and Qatar-1b
}

\author{
Ç. Püsküllü̈ ${ }^{\mathrm{a}, \mathrm{b}}$, F. Soydugan ${ }^{\mathrm{a}, \mathrm{b}}$, A.Erdem ${ }^{\mathrm{a}, \mathrm{b}}$, E.Budding $^{\mathrm{a}, \mathrm{c}}$ \\ ${ }^{a}$ Astrophysics Research Centre and Observatory, Çanakkale Onsekiz Mart University, Terzioğlu Kampüsü, \\ TR-17020, Çanakkale, Turkey \\ ${ }^{b}$ Department of Physics, Faculty of Arts and Sciences, Çanakkale Onsekiz Mart University, Terzioğlu \\ Kampüsü, TR-17020, Çanakkale, Turkey \\ ${ }^{c}$ Carter Observatory and SCPS and Victoria University of Wellington and Dept. Physics $\mathcal{E}$ Astronomy of \\ UoC, New Zealand
}

\begin{abstract}
New photometric follow-up observations of transitting 'hot Jupiters' TrES-3b and Qatar-1b are presented. Weighted mean values of the solutions of light curves in $R$-filter for both planetary systems are reported and compared with the previous results. The transit light curves were analysed using the WINFITTER code. The physical properties of the planets were estimated. The planet radii are found to be $R_{p}=1.381 \pm 0.033 R_{J}$ for TrES-3b and $R_{p}=$ $1.142 \pm 0.025 R_{J}$ for Qatar-1b. Transit times and their uncertainties were also determined and a new linear ephemeris was computed for both systems. Analysis of transit times showed that a significant signal could not be determined for TrES-3b, while weak evidence was found for Qatar-1b, which might be tested using more precise future transit times.
\end{abstract}

Keywords: eclipses - techniques: photometric - stars: planetary systems - stars: individual: TrES-3, stars: individual: Qatar-1

\section{Introduction}

The transit technique is one of the most powerful and effective methods for discovering exoplanets. The number of transiting exoplanets discovered has been increasing rapidly and has now exceeded one thousand Many of them are close-in gas giant planets also known as hot Jupiters. The basic astrophysical parameters of the exoplanets detected by this technique, and those of their host stars, can be derived with higher precision than those of exoplanets discovered by other methods (e.g. Southworth, 2008; Torres et al., 2008; Southworth, 2012; Ciceri et al., 2015). The data quality is important in this respect. The photometric data collected by the Kepler and CoRoT satellites has allowed us to discover

\footnotetext{
Email address: cpuskullu@comu.edu.tr (Ç. Püsküllü)

${ }^{1}$ Based on Transiting Extrasolar Planet Catalogue (TEPCat): http://www.astro.keele.ac.uk/jkt/tepcat
} 
many extrasolar planets and to derive the parameters of planet-star systems, precisely. In addition to space missions, ground-based photometric surveys keeping eyes on whole sky, play very important role in discovering new hot Jupiters. Follow up observations by the ground-based telescopes are publishing in the online database Exoplanet Transit Database (ETD) by many observers (Poddaný et al., 2010). This cumulated data make searching for additional planets in a system possible by using transit-timing variations (TTV). In this study, we focus on follow-up photometric transit observations and their analysis for hot Jupiters TrES-3b and Qatar-1b.

The TrES-3b system, discovered by O'Donovan et al. (2007), consists of a slightly metalpoor G-type star with a mass of $0.9 M_{\odot}$ and a hot Jupiter with an orbital period of 1.3 days. In their study, analysis of both photometric and radial velocity data was presented. The planet-star system, exhibiting a v-shape transit light curve, has interested many researchers (e.g. Gibson et al., 2009; Sozzetti et al., 2009; Colón et al., 2010; Southworth, 2011; Kundurthy et al., 2013; Vaňko et al., 2013). Sozzetti et al. (2009) re-determined the stellar parameters which are listed in Table 1 and stated that the precision and accuracy of basic planet properties may vary according to the methodologies used for analysis, as discussed by Torres et al. (2008); Rhodes and Budding (2014). Fressin et al. (2010) revealed that the orbit of TrES-3b is circular and ruled out tidal heating from the ongoing orbital circularization as an explanation for the inflated radius of TrES-3b. In order to search for additional planets in the system, transit timing variations based on follow-up observations were investigated in several studies (e.g. Sozzetti et al., 2009; Christiansen et al., 2011; Kundurthy et al., 2013, Vaňko et al., 2013). However, these authors could not find clear proof of the presence of a second planet in the system so far.

Qatar-1b has a mass of $1.09 M_{J}$. It was discovered by Alsubai et al. (2011). The planet is revolving around its parent star in a circular orbit with a period of 1.4 days. The host star is a slightly metal-rich K-type star with a mass of $0.85 M_{\odot}$. Its main properties are given in Table 1. Later, photometric observations and their analysis were reported by several authors (e.g. Maciejewski et al., 2015; Mislis et al., 2015; Collins et al., 2015). Covino et al. (2013) improved the orbital parameters and mentioned that the host star is moderately magnetodynamically active. A sinusoidal variation in the long-term light curves due to possible magnetic activity on the surface of the parent star was calculated by Mislis et al. (2015). First TTV analysis presented by von Essen et al. (2013) and they indicated a significant signal with a period of $\sim 190$ days. However more recently, Maciejewski et al. (2015) and Collins et al. (2015) could not find evidence of an additional planet in the Qatar1 bystem based on TTV analysis.

The main physical properties of the host star of TrES-3b and Qatar-1b are listed in Table 1. In this study, we present new transit light curves and their investigation for the exoplanets TrES-3b and Qatar-1b. Observations and reduction methods are given in Section 2. After showing parameter details emerging from the modelling, results of the solutions are reported in Section 3. The physical properties of the star-planet systems are then presented (Sect. 4), while updated ephemerides and TTV analysis are considered in Section 5. The final section summarizes and discusses the results for these hot Jupiters in particular, in the context of our general picture of hot Jupiter systems. 
Table 1: Basic parameters of the host stars of TrES-3b and Qatar-1b.

\begin{tabular}{lcc}
\hline Parameters & TrES-3 & Qatar-1 \\
\hline Mass, $M_{\star}\left(M_{\odot}\right)$ & $0.928_{-0.048}^{+0.028}$ & $0.838_{-0.041}^{+0.043}$ \\
Radius, $R_{\star}\left(R_{\odot}\right)$ & $0.829_{-0.022}^{+0.015}$ & $0.803 \pm 0.016$ \\
Density, $\rho_{\star}(c g s)$ & $2.304 \pm 0.066$ & $2.286_{-0.070}^{+0.074}$ \\
Surface gravity, $\log g_{\star}(c g s)$ & $4.4 \pm 0.1$ & $4.552_{-0.011}^{+0.012}$ \\
Effective temperature, $T_{e f f}(K)$ & $5650 \pm 75$ & $5013_{-88}^{+93}$ \\
{$[$ Fe $/ \mathrm{H}]($ dex $)$} & $-0.19 \pm 0.08$ & $0.171_{-0.094}^{+0.097}$ \\
Age $(\mathrm{Gyr})$ & $0.9_{-0.8}^{+2.8}$ & $1.19 \pm 0.47^{*}$ \\
References & Sozzetti et al. 2009 & Collins et al. 2015) \\
& & Mislis et al. 2015. \\
\hline
\end{tabular}

Table 2: Specifications of telescopes and CCD cameras used in study.

\begin{tabular}{lcc}
\hline Telescope and CCDs & Field of view $(\operatorname{arcmin})$ & Pixel size $\left(\operatorname{arcsec} \mathrm{px}^{-1}\right)$ \\
\hline T122 + AP42 & $7.8 \times 7.8$ & 0.23 \\
T122 + STL1001E & $7.1 \times 7.1$ & 0.42 \\
T60 + AP42 & $19.8 \times 19.8$ & 0.58 \\
T60 + STL1001E & $18 \times 18$ & 1.06 \\
T100 + SI1100 & $21.1 \times 20.8$ & 0.31 \\
\hline
\end{tabular}

\section{Observations and Data Reduction}

Our observations were made by three different-sized telescopes and camera configurations. The 122-cm Nasymth telescope (T122) and 60-cm German-Equatorial telescope (T60) are installed at Çanakkale Onsekiz Mart University Observatory (ÇOMUO) in Turkey. ÇOMUO has a $2048 \times 2048$ pixel Apogee Alta U42 CCD (AP42) camera and a $1024 \times 1024$ pixel SBIG STL 1001E CCD (STL1001E) camera which had been attached to the both telescopes various times. The other telescope (T100) used in the observations is installed at TÜBITAK National Observatory (TUG) in Antalya, Turkey. It has a primary mirror with 100-cm aperture size and is equipped with a SI 1100 CCD camera. Fields of view and pixel sizes of all telescopes and CCD cameras are listed in Table 2. Photometric data of TrES-3b transits were taken on six nights at T122, five nights at T100 and one night at T60, while those of Qatar-1b were obtained four nights each telescope. During the observations, Network Time Protocol (NTP) was used for time synchronization every minute. T100 has miliseconds accuracy in exposure time; however T122 and T60 have accuracies in seconds. Exposure times, CCD binning options and filters are given in Table 3 . Depending on weather conditions, many observations were also carried out using the defocusing method. Raw images were corrected with at least 10 biases, darks and flat images. Photometry was performed by the DAOPHOT package of IRAF 2 . We tried out different size apertures and ensured many comparison stars inside the CCD frame. The reduction process was completed using less scattered comparisons for both TrES-3b and Qatar-1b. Julian Date (JD UTC) was converted to Barycentric Julian Date (BJD $\mathrm{TDB}_{\mathrm{B}}$ ) (Eastman et al., 2010).

2 http://iraf.noao.edu/ 
Table 3: Journal of observations of hot Jupiters TrES-3b and Qatar-1b.

\begin{tabular}{|c|c|c|c|c|c|c|c|c|}
\hline Date & Telescope+CCD & Total Frame & Bin. & $\begin{array}{l}\text { Exp.Time } \\
(\mathrm{s})\end{array}$ & $\begin{array}{c}\text { Airmass } \\
\text { (Beginning - Ending) }\end{array}$ & $\begin{array}{c}\sigma_{0} \\
(\mathrm{mmag})\end{array}$ & $\beta$ & $\begin{array}{c}\sigma_{s} \\
(\mathrm{mmag})\end{array}$ \\
\hline \multicolumn{9}{|c|}{ TrES-3b } \\
\hline 03.06.2012 & $\mathrm{T} 122+\mathrm{AP} 42$ & 178 & 1 & 20 & $1.02-1.34$ & 4.7 & 3.2 & 15.2 \\
\hline 07.06.2012 & $\mathrm{T} 122+\mathrm{AP} 42$ & 109 & 1 & 20 & $1.02-1.09$ & 2.8 & 0.2 & 2.8 \\
\hline 27.05.2013 & $\mathrm{T} 122+\mathrm{AP} 42$ & 110 & 1 & 100 & $1.44-1.01$ & 2.3 & 0.4 & 2.3 \\
\hline 13.07.2013 & $\mathrm{T} 122+\mathrm{AP} 42$ & 123 & 1 & 60 & $1.02-2.55$ & 2.6 & 0.4 & 2.6 \\
\hline 15.06 .2014 & T100+SI1100 & 64 & 2 & 40 & $1.22-1.00$ & 3.4 & 0.3 & 3.4 \\
\hline 02.07 .2014 & $\mathrm{~T} 122+\mathrm{AP} 42$ & 216 & 2 & 45 & $1.00-1.08$ & 2.3 & 2.1 & 4.9 \\
\hline 27.04 .2015 & T100+SI1100 & 196 & 2 & 80 & $1.98-1.00$ & 2.4 & 0.9 & 2.6 \\
\hline 30.06 .2015 & T122+STL1001E & 97 & 1 & 80 & $1.02-1.32$ & 3.3 & 0.1 & 3.3 \\
\hline 08.07 .2015 & T100+SI1100 & 58 & 2 & 20 & $1.06-1.00$ & 2.5 & 0.1 & 2.5 \\
\hline 21.07.2015 & T100+SI1100 & 211 & 2 & 80 & $1.01-1.92$ & 1.9 & 1.4 & 2.8 \\
\hline 07.08 .2015 & T100+SI1100 & 154 & 2 & 80 & $1.00-1.43$ & 1.6 & 0.3 & 1.6 \\
\hline 24.08 .2015 & $\mathrm{~T} 60+\mathrm{AP} 42$ & 153 & 1 & 80 & $1.00-1.60$ & 2 & 0.9 & 3 \\
\hline \multicolumn{9}{|c|}{ Qatar-1b } \\
\hline 14.06.2014 & T100+SI1100 & 217 & 2 & 60 & $1.57-1.14$ & 2.5 & 0.6 & 2.5 \\
\hline 01.07 .2014 & $\mathrm{~T} 122+\mathrm{AP} 42$ & 195 & 1 & 80 & $1.40-1.20$ & 2.1 & 0.8 & 2.1 \\
\hline 28.07.2014 & $\mathrm{T} 122+\mathrm{AP} 42$ & 261 & 1 & 52 & $1.13-1.10-1.21$ & 2.2 & 0.6 & 2.2 \\
\hline 24.08 .2014 & $\mathrm{~T} 122+\mathrm{AP} 42$ & 269 & 1 & 100 & $1.12-1.10-1.64$ & 2.5 & 0.7 & 2.5 \\
\hline 20.09.2014 & T100+SI1100 & 245 & 2 & 90 & $1.14-1.14-2.37$ & 2.2 & 0.6 & 2.7 \\
\hline 30.09 .2014 & T60+STL1001E & 198 & 1 & 90 & $1.11-1.36$ & 2.6 & 0.9 & 2.6 \\
\hline 11.04 .2015 & T122+STL1001E & 120 & 1 & 120 & $2.19-1.25$ & 2.2 & 0.2 & 2.2 \\
\hline 15.05 .2015 & T100+SI1100 & 75 & 2 & 80 & $2.13-1.15$ & 3.4 & 0.1 & 3.4 \\
\hline 25.05 .2015 & T100+SI1100 & 226 & 2 & 80 & $1.30-1.16$ & 2.3 & 0.6 & 2.6 \\
\hline 04.08 .2015 & T122+STL1001E & 83 & 1 & 80 & $1.10-1.16$ & 2.1 & 1.2 & 2.1 \\
\hline 03.11 .2015 & T60+AP42 & 135 & 1 & 80 & $1.25-1.88$ & 2.5 & 0.4 & 2.5 \\
\hline 13.11.2015 & $\mathrm{T} 60+\mathrm{AP} 42$ & 149 & 1 & 80 & $1.19-1.78$ & 2.1 & 0.2 & 2.1 \\
\hline 20.11.2015 & T60+AP42 & 206 & 1 & 80 & $1.34-3.00$ & 4.4 & 0.5 & 4.4 \\
\hline
\end{tabular}




\section{Analysis of Transit Light Curves}

\subsection{Method}

In order to determine the physical and geometrical parameters of TrES-3b and Qatar-1b and their host stars, the WINFITTER program, which is basically adopted from the FITTER code, was used. FITTER was originally developed by Budding and Najim (1980) and refined by Budding and Zeilik (1987). The latest user-friendly design of the program with additional features was presented by Rhodes and Budding (2014) ${ }^{3}$. WINFITTER performs fitting optimization by means of a modified Marquardt-Levenberg application to analyse the light curves of eclipsing objects. The main physical parameters of the objects, namely, the masses $\left(M_{\star}\right.$ and $\left.M_{p}\right)$, radii $\left(R_{\star}\right.$ and $\left.R_{p}\right)$ and luminosities $\left(L_{\star}\right.$ and $\left.L_{p}\right)$ plus orbital parameters: period $(P)$, orbital inclination $(i)$ and eccentricity $(e)$, are used in the fitting process via a $\chi^{2}$-minimization computational algorithm. In the code, correlated errors are calculated from the diagonal of the inverted Hessian matrix. The FITTER code is based on the Radau model approach given by Kopal (1959). This approach gives tidal and rotational distortions (ellipticity), together with radiative interactions (reflection), of massive and relatively close gravitating bodies.

We followed same analysing procedure for all transit light curves of TrES-3b and Qatar-1b. During the analysis, the phase correction $\left(\Delta \phi_{0}\right)$, reference light level $(U)$, orbital inclination $(i)$, ratio of radii $\left(k=R_{p} / R_{\star}\right)$ and fractional radius of the host star $\left(r_{1}=R_{\star} / a\right.$ where $a$ is semi-major axis of the orbit) were taken as free parameters. We presented some of the calculated parameters, $r_{2}=R_{p} / a$ and total transit duration $T_{14}$ together with literature values in the Table 4. The fractional value of the secondary light contribution was set to zero under the assumption that the night side luminosity of the planet is essentially zero. The orbit was also assumed circular. We used the Claret and Bloemen (2011) tables to calculate the linear limb darkening coefficients: $u_{\text {TrES-3 }}(R)=0.567$ and $u_{\text {Qatar-1 }}(R)=0.668$. All transit curves were detrended by fitting a straight line to the out-of-transit data before analysing them in WINFITTER. Then, we calculated the standard deviation value $\left(\sigma_{0}\right)$ of this fit. In addition to $\sigma_{0}$, in order to reveal systematic effects (red noise) on the transit curves, the time-averaged detrending method, given by Winn et al. (2008), was used. As reported by Petrucci et al. (2015), the red noise factor is defined as $\beta=\sigma_{r} / \sigma_{N}$, where $\sigma_{r}$ is the standard deviation of binned residuals over $\mathrm{N}$ points. We selected the $\mathrm{N}$ numbers which represent $\mathrm{M}$ counts that were closer values to the division of the observation total duration by ingress/egress duration. Therefore, $\sigma_{N}$ is the expected deviation, as given by Eq. 1.

$$
\sigma_{N}=\frac{\sigma_{0}}{\sqrt{N}} \sqrt{\frac{M}{M-1}}
$$

Finally, the median of calculated $\beta$ values was used to scale $\sigma_{0}$ values to $\sigma_{s}$, which defines the final adopted data error, including underestimated noise (see Table 3).

\footnotetext{
${ }^{3}$ WINFITTER can be downloaded from the website: http://michaelrhodesbyu.weebly.com/astronomy.html
} 


\subsection{Solutions}

We collected 12 transit light curves for TrES-3b in the $R$-filter. The red noise factors for the data taken on 3 June 2012 and 2 July 2014, are $\beta=3.2$ and 2.1, respectively. Therefore, these two transit light curves were eliminated from the final weighted average values. The resulting parameters were obtained from WINFITTER, together with their errors, are listed in Table 4, including comparisons with previous studies. Theoretical curves and their agreement with observational data can be seen in Fig. 1. The TrES-3b transit model curves were found to be in the range, $\chi_{\text {red }}^{2}=0.8-1.2$ over our first estimated error, $\sigma_{0}$. Hence, we cannot say definitely that there may be additional physical effects, such as maculation, i.e. spots on the surface of the host star. The ratio of percentage change of $r_{1}$ is $15 \%$, from 0.15 to 0.18 ; for $k$ is $21 \%$, and for $i$ is $1 \%$, when errors are excluded.

We obtained 12 transit light curves in the $R$-filter for Qatar-1b. The light curve observed on 20th November 2015 was not used in the analysis since it had large scatter, $\sigma_{0}=4.4$ However, the transit time was calculated for these data and evaluated for transit time analysis, as presented in Section 5. The highest red noise factor for the 11 light curves was 1.2. Weighted mean values of the resulting parameters are given in Table 5 together with errors, and also those presented in previous studies. Figure 2 shows the agreement between the theoretical curves and transit data for Qatar-1b. Since the our data scattering of Qatar-1b was lower than TrES-3b, fitting results were also derived inside a narrow parameter space which is mostly spread around $10 \%$ for individual parameters.

Lastly, the mean system geometries were constructed using binned data and a model calculated from the weighted mean values, as seen in Fig. 3, for the star-planet systems TrES-3b and Qatar-1b. In order to check for limb darkening values, the binned transit light curves were solved taking limb darkening parameters as free. These were obtained as $u_{\text {TrES-3 }}=0.62 \pm 0.03$ and $u_{\text {Qatar- } 1}=0.67 \pm 0.03$. These results may be compared with the theoretical values of $\left(u_{\text {TrES-3 }}=0.567\right.$ and $\left.u_{\text {Qatar- } 1}=0.668\right)$ by Claret and Bloemen $(2011)$.

\section{Physical Properties}

In order to determine the astrophysical parameters of the hot Jupiters, TrES-3b and Qatar-1b, the photometric results in Table 5 together with basic parameters given in Table 1 were used. The mass of planet and its host star in the Qatar-1 system were taken from Alsubai et al. (2011) while the mass values of TrES-3b and its host star were used as given by Sozzetti et al. (2009) and Torres et al. (2008), respectively. We calculated the physical parameters of the stars $\left(R_{\star}, \rho_{\star}, \log g_{\star}, T_{\text {eff }}\right)$ and planets $\left(R_{p}, \rho_{p}, \log g_{p}, T^{\prime}{ }_{e q}\right)$ from the weighted mean values of all transit parameters. These results with standard errors are given in Table 6. A comparison between the binned and combined transit data, and theoretical light curves were calculated from the weighted mean parameters listed in Tables 4 and 5 , can be seen in Fig. 3 for TrES-3b and Qatar-1b, respectively. Binning was done to 70 points for each transit curve. Transit geometries are also plotted with the corresponding curves (see upper parts of the Figures 3). 


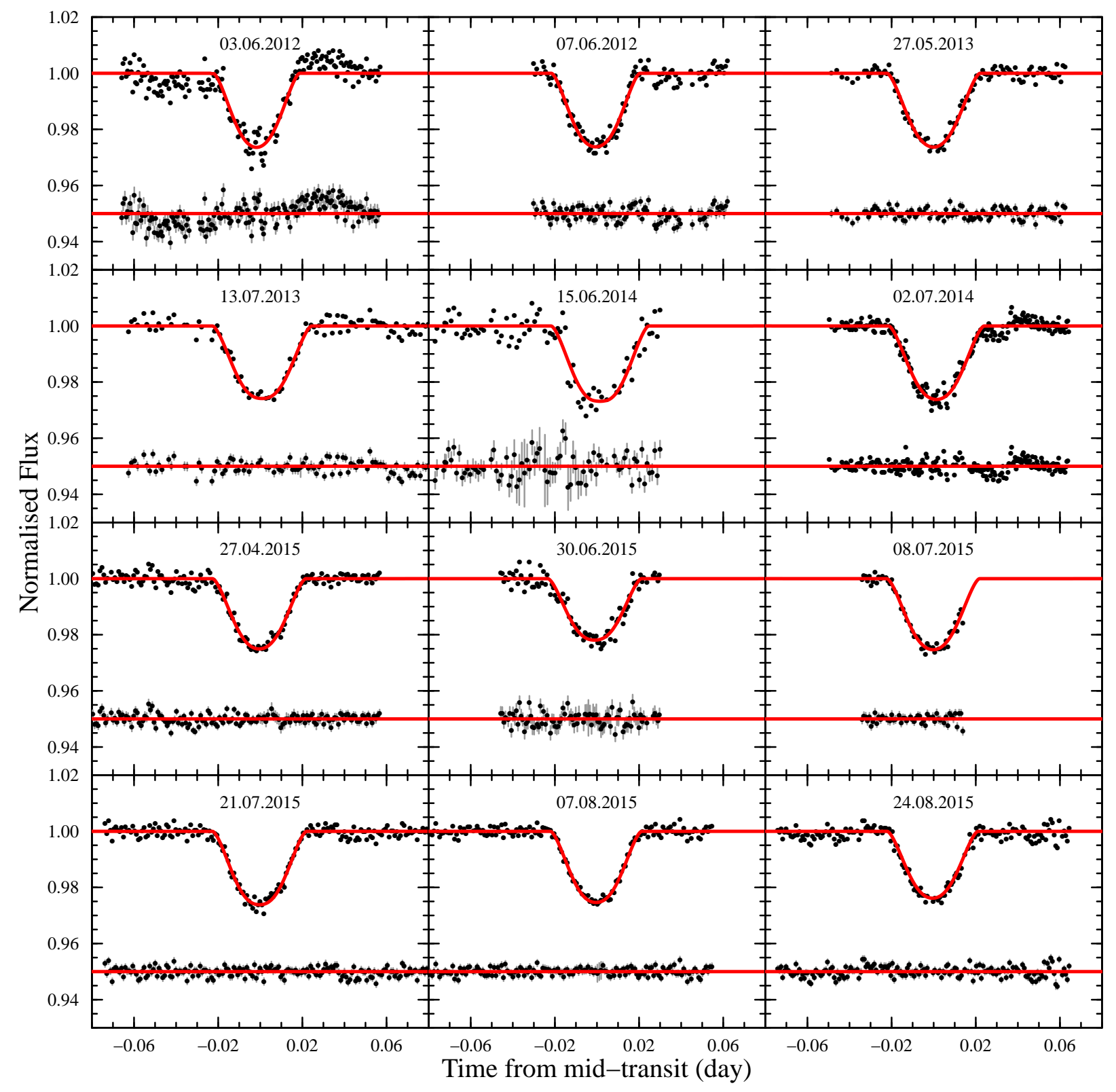

Figure 1: Modelled individual transit curves of TrES-3b in $R$-filter with photometric error bars and residuals from fits. 


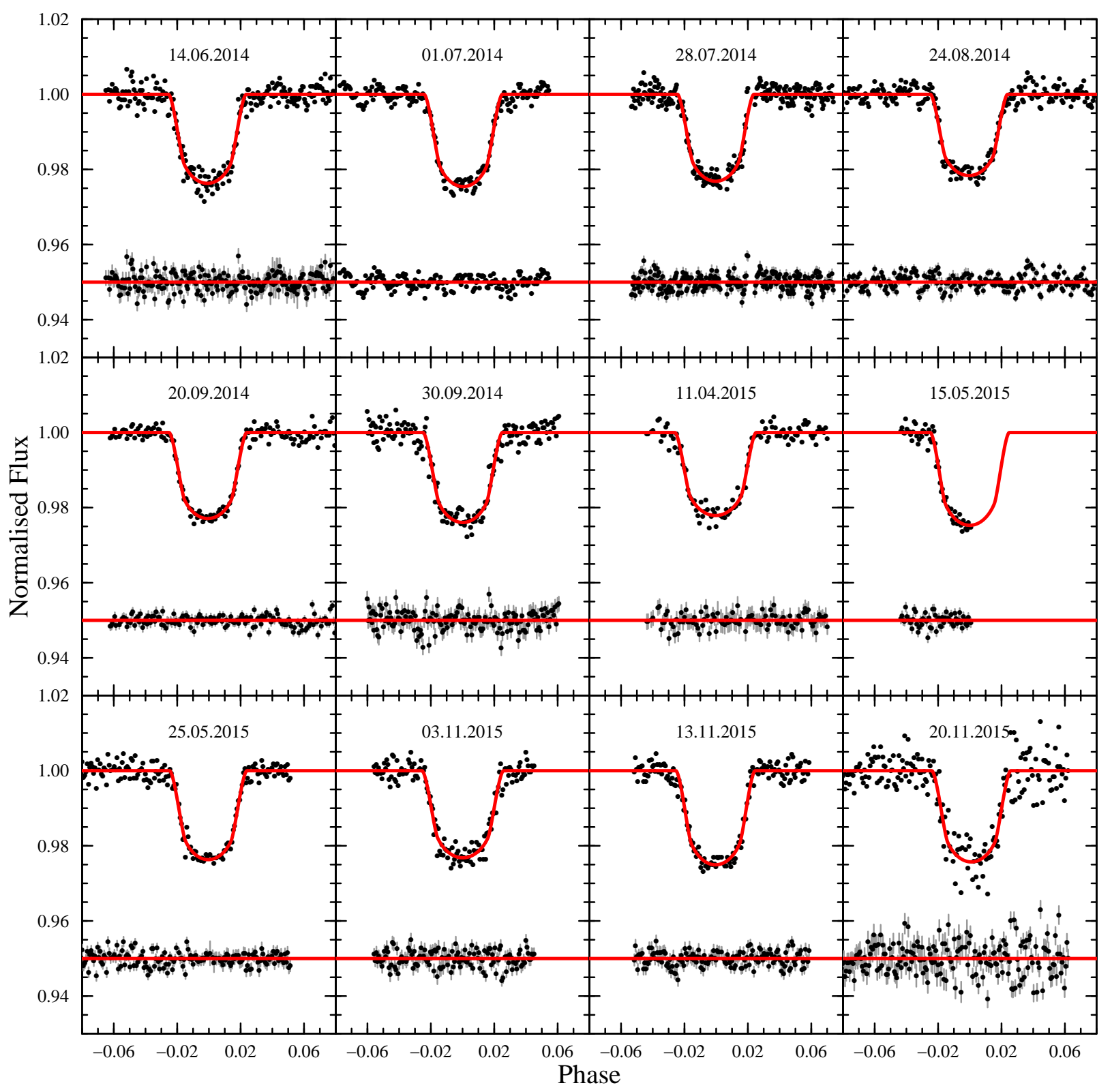

Figure 2: Modelled individual transit curves of Qatar-1b in $R$ - filter with photometric error bars and residuals from fits. 
Table 4: Fitting model parameters of TrES-3b comparing with previous studies. First column is listing references, following columns are the fractional radius of host star $r_{1}$, ratio of radii $(k)$, orbital inclination (i) and total transit time $\left(T_{14}\right)$ respectively.

\begin{tabular}{|c|c|c|c|c|}
\hline References & $r_{1}$ & $k\left(=r_{2} / r_{1}\right)$ & $i(\operatorname{deg})$ & $T_{14}(\min )$ \\
\hline O'Donovan et al. (2007) & $0.1650 \pm 0.0027$ & $0.1660 \pm 0.0024$ & $82.15 \pm 0.21$ & - \\
\hline Sozzetti et al. $(2009)$ & $0.1687 \pm 0.0140$ & $0.1655 \pm 0.0020$ & $81.85 \pm 0.16$ & - \\
\hline Gibson et al. (2009) & - & $0.1664 \pm 0.0011$ & $81.73 \pm 0.13$ & $79.92 \pm 1.44$ \\
\hline Colón et al. (2010) & - & $0.1662 \pm 0.0046$ & - & $83.77 \pm 1.15$ \\
\hline Southworth $(2010)^{\mathrm{a}}$ & $0.1666 \pm 0.0017$ & $0.1639 \pm 0.0037$ & $82.07 \pm 0.17$ & - \\
\hline Lee et al. $(2011)$ & $0.1674 \pm 0.0023$ & $0.1603 \pm 0.0042$ & $81.77 \pm 0.14$ & - \\
\hline Christiansen et al. (2011) & $0.1664 \pm 0.0204$ & $0.1661 \pm 0.0343$ & $81.99 \pm 0.30$ & $81.9 \pm 1.10$ \\
\hline Southworth $(2011)^{\mathrm{a}}$ & $0.1682 \pm 0.0014$ & $0.1635 \pm 0.0025$ & $81.93 \pm 0.13$ & - \\
\hline \multirow{2}{*}{ Kundurthy et al. 2013$)^{\mathrm{b}}$} & $0.1675 \pm 0.0008$ & $0.1652 \pm 0.0009$ & $81.95 \pm 0.06$ & - \\
\hline & $0.1698 \pm 0.0014$ & $0.1649 \pm 0.0015$ & $81.51 \pm 0.14$ & - \\
\hline Turner et al. (2013) & $0.1721 \pm 0.0054$ & $0.1693 \pm 0.0087$ & $81.35 \pm 0.63$ & $81.3 \pm 0.23$ \\
\hline \multirow{2}{*}{ Vaňko et al. $(2013)^{\text {b }}$} & $0.1682 \pm 0.0032$ & $0.1644 \pm 0.0047$ & $81.86 \pm 0.28$ & $79.2 \pm 1.38$ \\
\hline & $0.1696 \pm 0.0024$ & $0.1669 \pm 0.0027$ & $81.76 \pm 0.14$ & $79.08 \pm 0.72$ \\
\hline This study & $0.1691 \pm 0.0024$ & $0.1709 \pm 0.0030$ & $81.83 \pm 0.21$ & $83.98 \pm 0.75$ \\
\hline
\end{tabular}

${ }^{a}$ present solutions obtained from light curves in the literature.

b present two solutions estimated using different methods.

Table 5: Fitting model parameters of Qatar-1b comparing with previous studies. The column parameters are same as in the Table 4 .

\begin{tabular}{|c|c|c|c|c|}
\hline References & $r_{1}$ & $k\left(=r_{2} / r_{1}\right)$ & $i(\operatorname{deg})$ & $T_{14}(\min )$ \\
\hline Alsubai et al. (2011) & $.1633 \pm 0.0053$ & $0.1454 \pm 0.0015$ & $83.47 \pm 0.40$ & $96.71 \pm 1.11$ \\
\hline von Essen et al. (2013) & $0.1558 \pm 0.0024$ & $0.1435 \pm 0.0008$ & $84.52 \pm 0.24$ & - \\
\hline Covino et al. $(2013)$ & $0.1601 \pm$ & 0.151 & $83.82 \pm$ & $97.63 \pm 1.44$ \\
\hline Mislis et al. (2015) & $0.1640 \pm$ & 0.147 & $84.03 \pm$ & - \\
\hline Maciejewski et al. (2015) & $0.1582 \pm$ & $0.1459 \pm$ & 84.26 & $98.50=$ \\
\hline Collins et al. $(2015)$ & & $0.1463 \pm$ & & $99.66 \pm 0.47$ \\
\hline This study & $0.1584 \pm 0.0031$ & $0.1470 \pm 0.0012$ & $84.42 \pm 0.30$ & $100.84 \pm 0.92$ \\
\hline
\end{tabular}



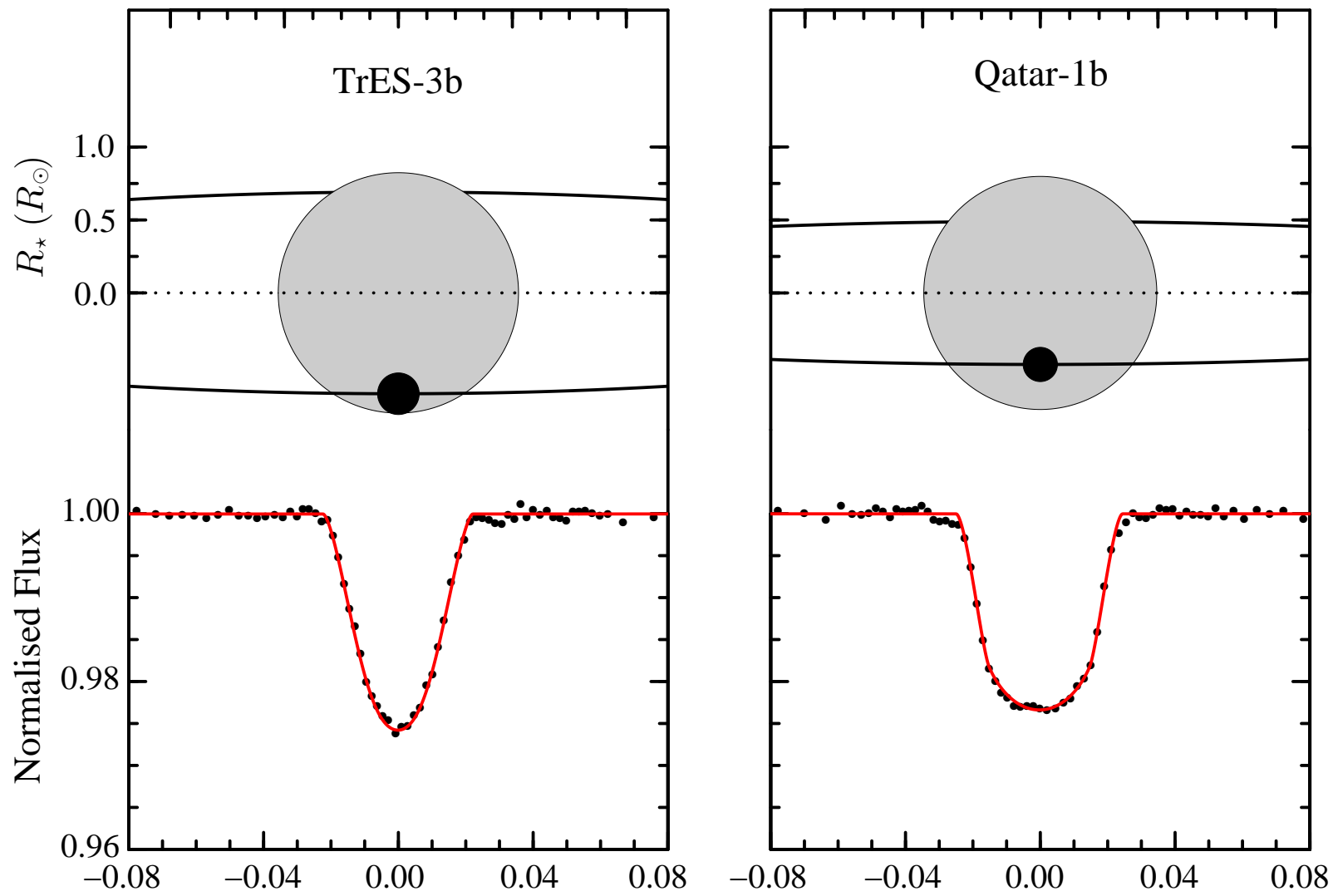

\section{Phase}

Figure 3: System geometries and transit light curves at zero point phase both TrES-3b (left panel) and Qatar1b (right panel). The observational points indicate combined transit light curve of the planet for all normalized individual transit curves binned to 70 points. The continuous line shows the theoretical curve were calculated using the weighted mean solution. 


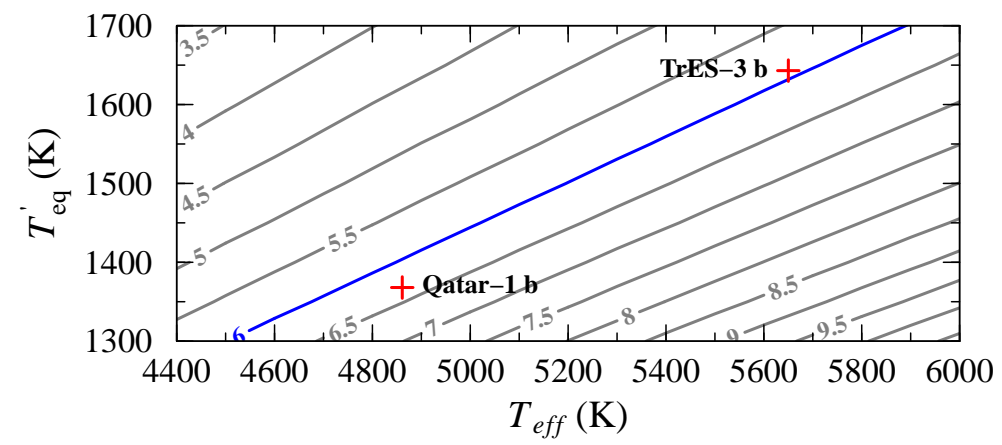

Figure 4: Temperature parameters, $T_{\text {eff }}$ and $T_{e q}^{\prime}$ plotted together in order to view diversity between flux received on planets by their host stars. Solid lines indicate $a / R_{\star}$ steps. The planets are labelled with crossmarks. Both planets, as expected, appear on same line, of $a / R_{\star} \sim 6$, coloured blue.

As a measure of gravitational focusing, the Safronov number $(\Theta)$ given by Hansen and Barman (2007) is

$$
\Theta=\frac{a}{R_{p}} \frac{M_{p}}{M_{\star}} .
$$

They proposed a classification for exoplanets using the values of the Safranov number. According to this designation, exoplanets with $\Theta \sim 0.07 \pm 0.01$ were termed Class I, while those having $\Theta \sim 0.04 \pm 0.01$ were Class II. While we calculated $\Theta_{\text {TrES-3 b }}=0.068 \pm 0.003$ for TrES-3b, meaning that it falls within the Class I group, Qatar-1b appears to be between Class I and Class II, with $\Theta_{\text {Qatar-1b }}=0.053 \pm 0.002$.

The equilibrium temperature was also estimated by the simplified equation given by Southworth (2010):

$$
T_{e q}^{\prime}=T_{e f f} \sqrt{\frac{R_{\star}}{2 a}}
$$

where $a$ is in same unit with stellar radius, $R_{\star}$. The effective temperature of the host stars, $T_{\text {eff }}$ were obtained from Torres et al. (2008) and Alsubai et al. (2011) for TrES-3b and Qatar-1b, respectively. Due to the similar-size orbit and almost identical $R_{\star}$ values, the ratio $T_{e q}^{\prime} / T_{\text {eff }}$ should be approximately the same for these two exoplanets. In order to illustrate this similarity, we plotted $T_{e f f}$ against $T_{e q}^{\prime}$ with the semi-major axis in stellar radii, $a / R_{\star}$ contour lines in Fig. 4. As expected, both planets lie on the same $a / R_{\star}$ contour line.

\section{Transit Ephemeris and Transit Timing Variations}

The investigation of additional bodies or unseen components around binary stars is a common research area in stellar astrophysics. In particular, the O-C method based on eclipse times is frequently used for this purpose. The method is also preferred when searching for additional planets around star-planet systems. In the field of multi-planet research, very precise transit times at least in several tens seconds of accuracy are required since the expected amplitudes in the O-C diagrams are very low (e.g. Lithwick et al., 2012). 
Table 6: Astrophysical parameters of star-planet systems TrES-3b and Qatar-1b.

\begin{tabular}{lcc}
\hline Parameters & TrES-3b & Qatar-1b \\
\hline Adopted values & & \\
Stellar mass, $M_{\star}\left(M_{\odot}\right)$ & $0.928 \pm 0.031$ & $0.850 \pm 0.030$ \\
Planet mass, $M_{p}\left(M_{J}\right)$ & $1.910 \pm 0.065$ & $1.090 \pm 0.080$ \\
Effective temperature of the host star, $T_{\text {eff }}(K)$ & $5650 \pm 75$ & $4861 \pm 125$ \\
Derived values & & \\
Semi-major axis, $a / R_{\star}$ & $5.92 \pm 0.11$ & $6.31 \pm 0.15$ \\
Stellar radius, $R_{\star}\left(R_{\odot}\right)$ & $0.826 \pm 0.012$ & $0.798 \pm 0.016$ \\
Stellar density, $\rho_{\star}\left(\rho_{\odot}\right)$ & $1.607 \pm 0.074$ & $1.666 \pm 0.103$ \\
Stellar surface gravity, $\operatorname{logg}_{\star}(\mathrm{cgs})$ & $4.57 \pm 0.01$ & $4.56 \pm 0.02$ \\
Planet radius, $R_{p}\left(R_{J}\right)$ & $1.381 \pm 0.033$ & $1.142 \pm 0.025$ \\
Planet density, $\rho_{p}\left(\rho_{J}\right)$ & $0.668 \pm 0.049$ & $0.679 \pm 0.045$ \\
Planet surface gravity, $\operatorname{logg}_{p}(\mathrm{cgs})$ & $3.39 \pm 0.02$ & $3.32 \pm 0.02$ \\
Equilibrium temperature of the planet, $T_{e q}^{\prime}(K)$ & $1643 \pm 25$ & $1368 \pm 30$ \\
Safronov Number, $\Theta$ & $0.068 \pm 0.003$ & $0.053 \pm 0.002$ \\
Incident flux, $\langle F\rangle\left(10^{9} \mathrm{ergs}^{-1} \mathrm{~cm}^{-2}\right)$ & $1.650 \pm 0.079$ & $0.798 \pm 0.060$ \\
\hline
\end{tabular}

In this study, we also used the $\mathrm{O}-\mathrm{C}$ method to analyse transit times of the target hot Jupiters. As a first step, mid-transit times from WINFITTER solutions for both the TrES-3b and Qatar-1b systems were determined. The code calculates a $\Delta \phi_{0}$ parameter, which was later converted to transit time in BJD. We also supplemented our data by retrieving values having errors lower than 0.0005 BJD (43.2 s) from literature and the ETD, selecting clear transit curves. In this study, 12 mid-transit times for TrES-3b and 11 mid-transit times for Qatar-1b were obtained and are listed in Table 7. Epoch numbers and O-C values in Table7 were calculated using the new transit ephemeris in Eqs. 4 and 5, which led to improvements for each object, as presented below. We generated Lomb-Scargle periodograms (Lomb, 1976, Scargle, 1982) inside frequency windows limited to the Nyquist frequency with the improved TTVs. The false alarm probability (FAP) was also checked to ensure that the detected dominant frequencies were significant (Horne and Baliunas, 1986).

\section{1. $\operatorname{TrES}-3 b$}

New light elements of the TrES-3b were determined using our transit times together with data from the ETD and times given by Sozzetti et al. (2009); Gibson et al. (2009); Lee et al. (2011); Christiansen et al. (2011); Kundurthy et al. (2013); Turner et al. (2013) and Vaňko et al. (2013). An improved ephemeris was calculated as follows:

$$
T_{c}=(B J D) 2456082.49390(3)+1.30618652(4) \times E
$$

where $\mathrm{E}$ is the epoch number. Using the weighted linear fits to the $\mathrm{O}-\mathrm{C}$ data, we obtained a reduced chi-squared value, $\chi_{\text {red }}^{2}=7.8$. Despite this high value of $\chi_{\text {red }}^{2}$, no peaks in our Lomb-Scargle analyses for TrES-3b exceeded $5 \%$ of the significance level. Since no clear dominant peak was observable; we would like to present the highest peak at a 
Table 7: Mid-transit times of TrES-3b and Qatar-1b.

\begin{tabular}{|c|c|c|c|c|}
\hline Date & Filter & Epoch & $T_{0}(\mathrm{BJD} 2450000+)$ & $\mathrm{O}-\mathrm{C}$ (days) \\
\hline \multicolumn{5}{|c|}{ TrES-3b } \\
\hline 03.06 .2012 & $R_{\text {Bessell }}$ & 0 & $6082.49611 \pm 0.00056$ & 0.00221 \\
\hline 07.06 .2012 & $R_{\text {Bessell }}$ & 3 & $6086.41313 \pm 0.00034$ & 0.00067 \\
\hline 27.05.2013 & $R_{\text {Bessell }}$ & 274 & $6440.38897 \pm 0.00028$ & -0.00004 \\
\hline 13.07.2013 & $R_{\text {Bessell }}$ & 310 & $6487.41045 \pm 0.00043$ & -0.00127 \\
\hline 15.06.2014 & $R_{\text {Bessell }}$ & 568 & $6824.40585 \pm 0.00064$ & -0.00198 \\
\hline 02.07.2014 & $R_{\text {Bessell }}$ & 581 & $6841.38641 \pm 0.00030$ & -0.00185 \\
\hline 27.04 .2015 & $R_{\text {Bessel }}$ & 810 & $7140.50549 \pm 0.00033$ & 0.00052 \\
\hline 30.06 .2015 & $R_{\text {Cousins }}$ & 859 & $7204.50938 \pm 0.00044$ & 0.00126 \\
\hline 08.07 .2015 & $R_{\text {Bessell }}$ & 865 & $7212.34536 \pm 0.00037$ & 0.00013 \\
\hline 21.07.2015 & $R_{\text {Bessell }}$ & 875 & $7225.40740 \pm 0.00030$ & 0.00030 \\
\hline 07.08 .2015 & $R_{\text {Bessell }}$ & 888 & $7242.38793 \pm 0.00028$ & 0.00040 \\
\hline 24.08.2015 & $R_{\text {Bessell }}$ & 901 & $7259.36845 \pm 0.00034$ & 0.00050 \\
\hline \multicolumn{5}{|c|}{ Qatar-1b } \\
\hline 14.06.2014 & $R_{\text {Bessell }}$ & 0 & $6823.41500 \pm 0.00044$ & 0.00138 \\
\hline 01.07.2014 & $R_{\text {Bessell }}$ & 12 & $6840.45327 \pm 0.00042$ & -0.00065 \\
\hline 28.07.2014 & $R_{\text {Bessell }}$ & 31 & $6867.43480 \pm 0.00042$ & 0.00041 \\
\hline 24.08.2014 & $R_{\text {Bessell }}$ & 50 & $6894.41548 \pm 0.00047$ & 0.00063 \\
\hline 20.09.2014 & $R_{\text {Bessell }}$ & 69 & $6921.39618 \pm 0.00048$ & 0.00085 \\
\hline 30.09.2014 & $R_{\text {Cousins }}$ & 76 & $6931.33528 \pm 0.00053$ & -0.00022 \\
\hline 11.04.2015 & $R_{\text {Cousins }}$ & 212 & $7124.45935 \pm 0.00059$ & 0.00047 \\
\hline 25.05 .2015 & $R_{\text {Bessell }}$ & 243 & $7168.48014 \pm 0.00058$ & 0.00050 \\
\hline 03.11.2015 & $R_{\text {Bessell }}$ & 357 & $7330.36216 \pm 0.00065$ & -0.00030 \\
\hline 13.11.2015 & $R_{\text {Bessell }}$ & 364 & $7340.30315 \pm 0.00064$ & 0.00051 \\
\hline 20.11.2015 & $R_{\text {Bessell }}$ & 369 & $7347.40134 \pm 0.00085$ & -0.00142 \\
\hline
\end{tabular}




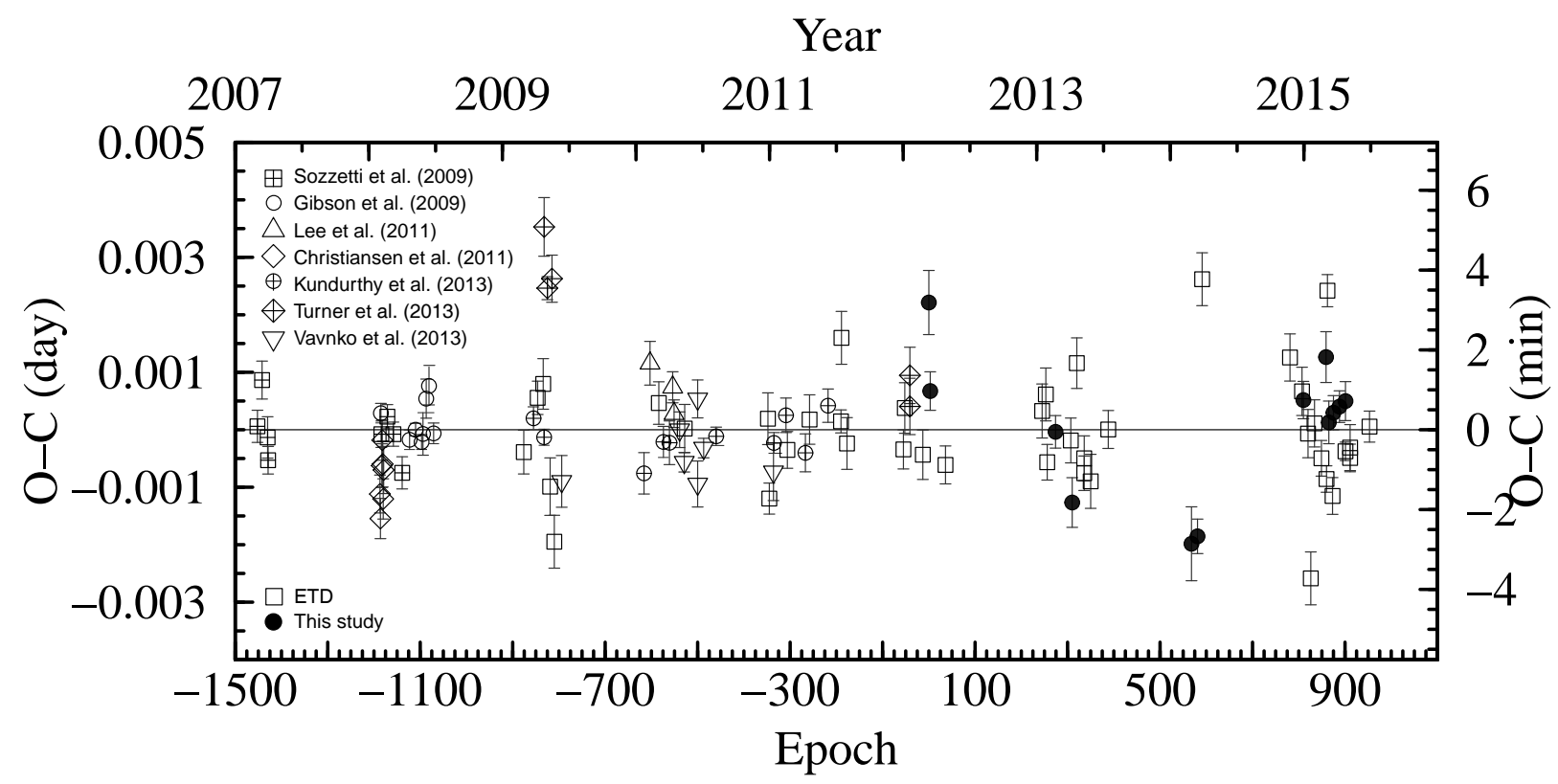

Figure 5: O-C diagram for TrES-3b.

frequency of $\nu_{1 \text { (TrES-3 } \mathrm{b})}=0.0154 \pm 0.0001$ cycl $P^{-1}$, which corresponds to a TTV period of $P_{t t v}=84.79 \pm 0.35$ days. The O-C diagram of TrES-3b can be seen in Fig. 5 .

\subsection{Qatar-1b}

We used transit times for Qatar-1b was by combining our mid-transit times with data from von Essen et al. (2013), Maciejewski et al. (2015) and ETD. The improved linear ephemeris obtained is as follows:

$$
T_{c}=(B J D) 2456823.41361(4)+1.4200248(1) \times E
$$

By applying linear least squares fits, a reduced $\chi_{\text {red }}^{2}=4.5$ was arrived at. The corresponding Lomb-Scargle periodogram shows a few strong peaks, but they are all under $5 \%$ significance level. The highest peak is seen at the frequency $\nu_{1 \text { (Qatar-1b) }}=0.0169 \pm 0.0001$ cycl $P^{-1}$, which corresponds to $P_{t t v}=83.75 \pm 0.48$ days (see Fig. 6). We obtained a value of $\chi_{\text {red }}^{2}=3.9$ after applying a least squares sinusoidal fit to the O-C data with a derived amplitude of $A_{t t v}=0.00044 \pm 0.00011$ days $(38.0 \pm 9.5 \mathrm{~s})$. In Fig. 7, the distribution of the O-C data together with its sinusoidal TTV can be seen. The parameters of this sinusoidal variation were found to be similar to those determined in von Essen et al. (2013).

\section{Discussion and Conclusions}

This study has given a photometric investigation of the transiting exoplanets TrES-3b and Qatar-1b. The transit light curves covering or partly covering the transit phases for both planets, were used to determine their photometric parameters, which were found to 


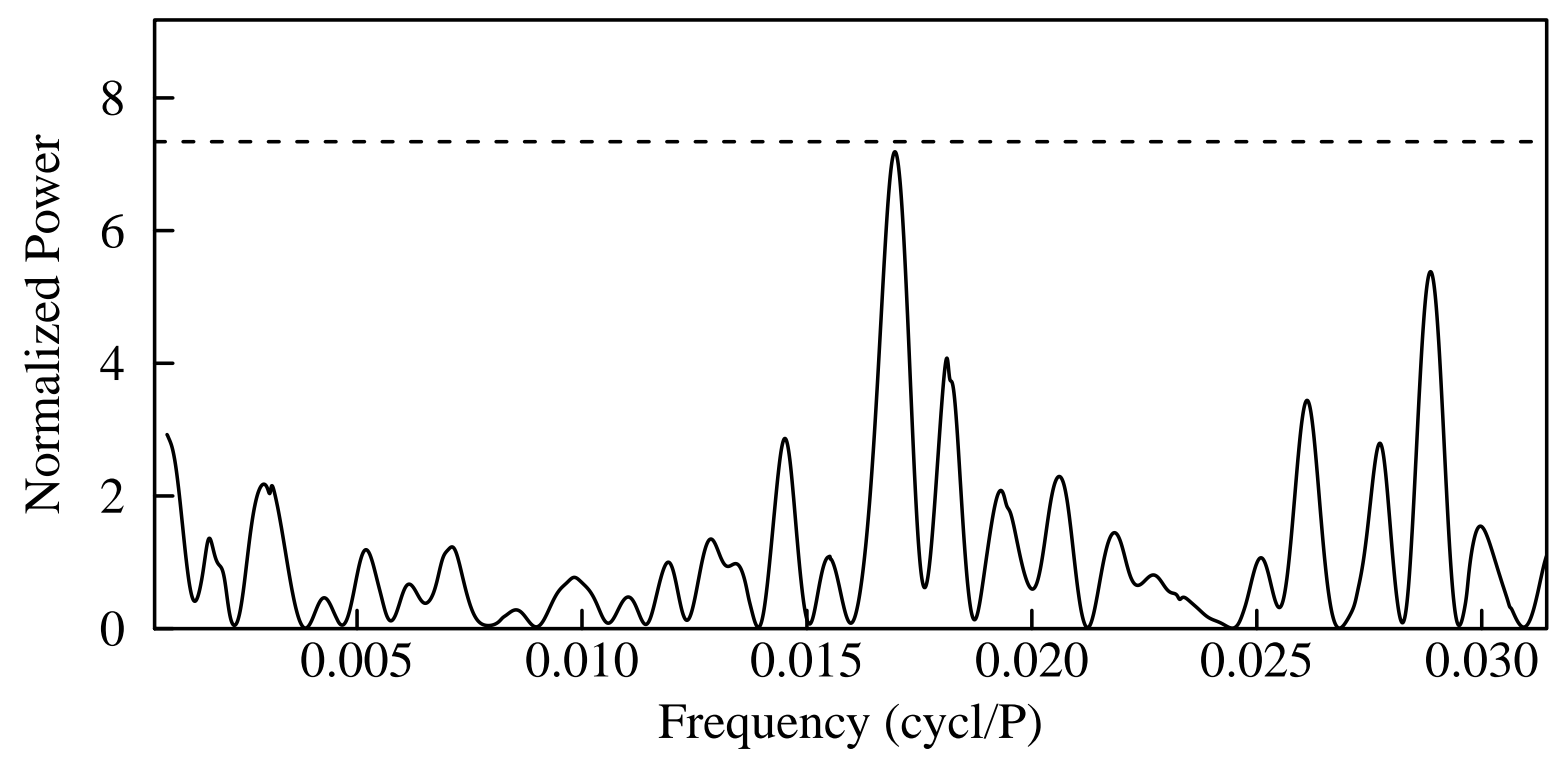

Figure 6: Lomb-Scargle periodogram of O-C for Qatar-1b. Dashed line shows 5\% significance level.

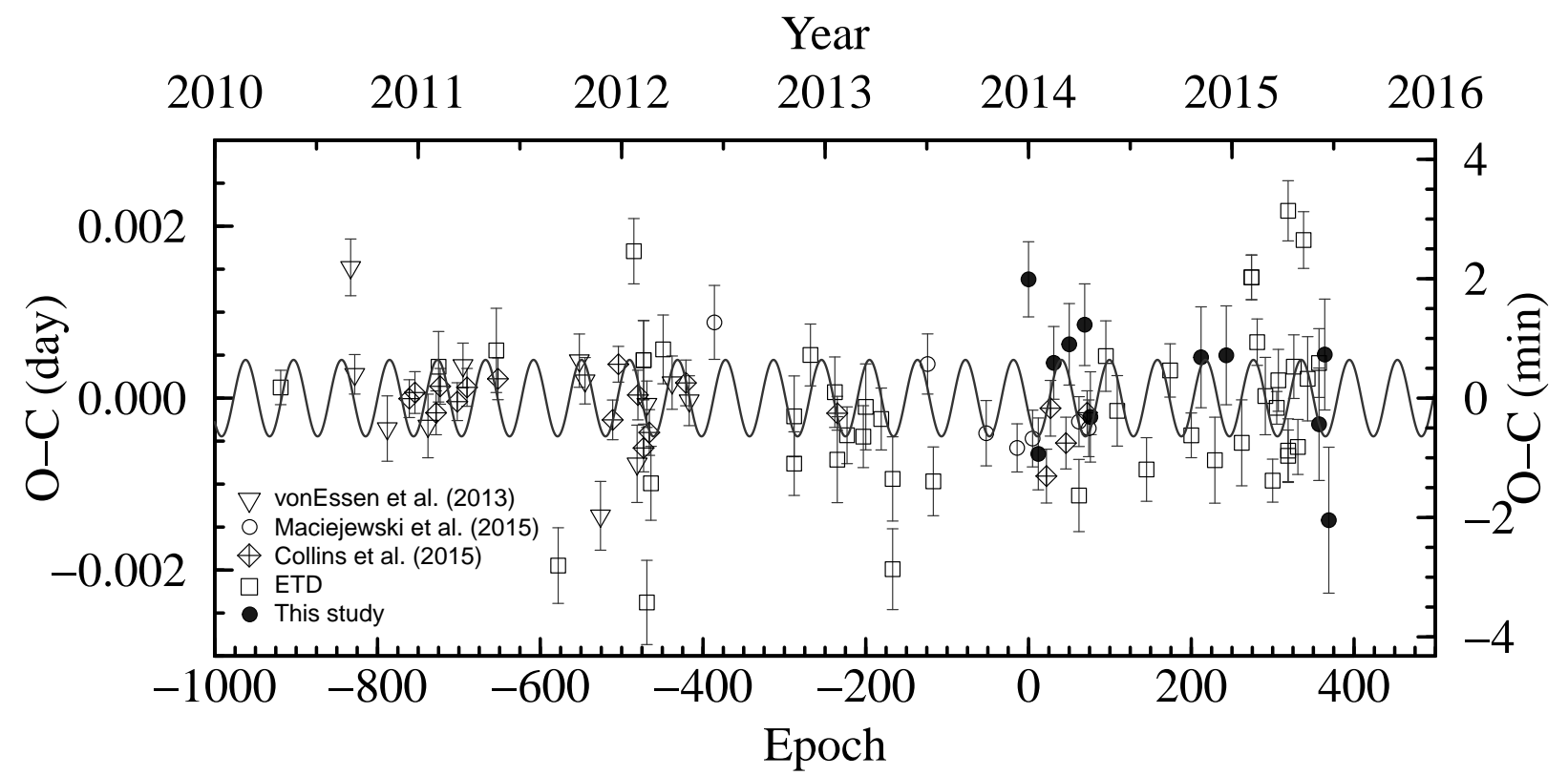

Figure 7: O-C diagram for Qatar-1b. Applied least square fit to sinusoidal variation with obtained a reduced $\chi_{\text {red }}^{2}=3.9$, is indicated by the continuous line. 
be generally consistent with previous results. It is of interest to examine these exoplanets together and compare their parameters, since they have approximately similar size orbits.

We found a radius of $R_{p}=1.381 \pm 0.033 R_{J}$ for TrES-3b and $R_{p}=1.142 \pm 0.025 R_{J}$ for Qatar-1b. Although there are similarities in the orbits of the planets, these planets and their host stars have different physical properties (see Fig. 8). The most conspicuous differences are in the masses of the planets and the metallicities of their host stars. The mass of TrES-3b is $1.91 M_{J}$ while that of Qatar-1b is $1.09 M_{J}$. These differences put the planets in separate classes when defined by their Safronov numbers. The more massive planet TrES-3b is a Class I planet with $\Theta_{\text {TrES-3b }}=0.068 \pm 0.002$, while Qatar-1b is a Class II planet with $\Theta_{\text {Qatar- } 1 \mathrm{~b}}=0.053 \pm 0.002$.

The transit times and their uncertainties were determined and analysed for possible transit time variations (TTVs). We obtained 12 mid-transit times for TrES-3b and 11 mid-transit times for Qatar-1b, with uncertainties in the range of 24-70 seconds. The linear ephemeris for both systems was improved. We couldn't determine any significant signal from O-C times of TrES-3b; however nearly clear periodicity of Qatar-1b TTV was obtained at a $6.5 \%$ significance level compared with the FAP value of $28 \%$ for the strongest peak given by Maciejewski et al. (2015). von Essen et al. (2013) reported TTV periods of $187 \pm 17$ and $386 \pm 54$ days with a fitted amplitude of $A_{t t v}=0.00052 \pm 0.00020$ for first peak. These values are propotional with our TTV period of $83.75 \pm 0.48$ days. We estimated a TTV amplitude of $A_{t t v}=0.00044 \pm 0.00011$. This results give us support to result in that there is a perturbing object in the system. After sinusoidal fit to O-C, we improved $\chi_{\text {red }}^{2}$ from 4.5 to 3.9. The reason that we still have a high $\chi_{r e d}^{2}$ value, is that the data is spreading out a period of 8 years with $\sigma_{O-C}=69.1$ seconds. We had lowest individual points error of $\sigma_{O}=16.4$ seconds in O-C. At these conditions, to check the existence of new planets in the system, more precise transit times spread over time are needed. As given in the first paragraph of Section 5, time accuracy should be in the order of several tens of seconds at least and data should spread over time in order to discover additional planets in star-planet systems (e.g. Lithwick et al., 2012).

We collected data from the TEPCat catalogue for exoplanets having orbital radii smaller than $a=0.024$ AU to compare planets in general with our targets. As seen in Fig. 8, WASP-46b has a similar orbit size and temperature to that of TrES-3b, while there are only two known exoplanets, Qatar-2b and OGLE-TR-113b, with similar temperatures, which are closer than Qatar-1b to their host stars. From the mass-radius relation of the planets presented in Fig. 9, one of our massive planets, TrES-3b, remains near the $\rho=2.0 \rho_{J}$ density curve, and appears rather exceptional in that respect. Qatar-1b, on the other hand, is more similar to Jupiter.

The stellar metallicities, $[\mathrm{Fe} / \mathrm{H}]_{\text {TrES-3 }}=-0.2<[\mathrm{Fe} / \mathrm{H}]_{\text {Qatar- } 1}=0.2$ have raised some questions about the structure of the corresponding planets and their heating mechanisms in the literature (Torres et al., 2008; Alsubai et al., 2011). The host star of TrES-3b may have magnetic activity, as mentioned by Christiansen et al. (2011). While there is no signal of this in our data, it might be investigated again appending much more precise observations extending further in time. 


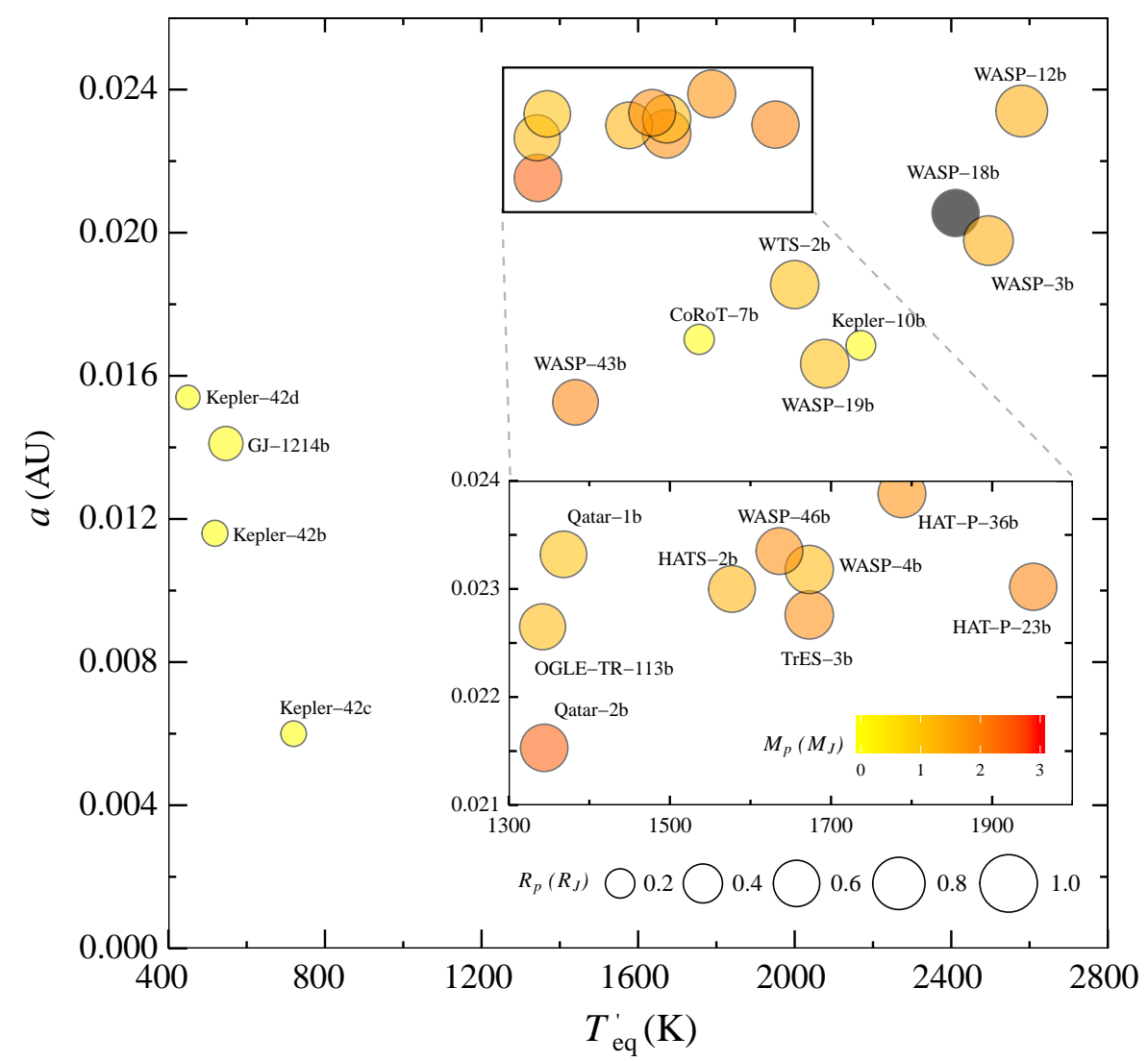

Figure 8: Location of exoplanets having orbit size $(a)$ values smaller than $0.024 \mathrm{AU}$ in plane of $T_{e q}^{\prime}-a$ diagram. Data except for TrES-3b and Qatar-1b taken from TEPCat database. The super massive $\left(M_{p}=\right.$ $10.5 M_{J}$ ) planet WASP-18b coloured grey.

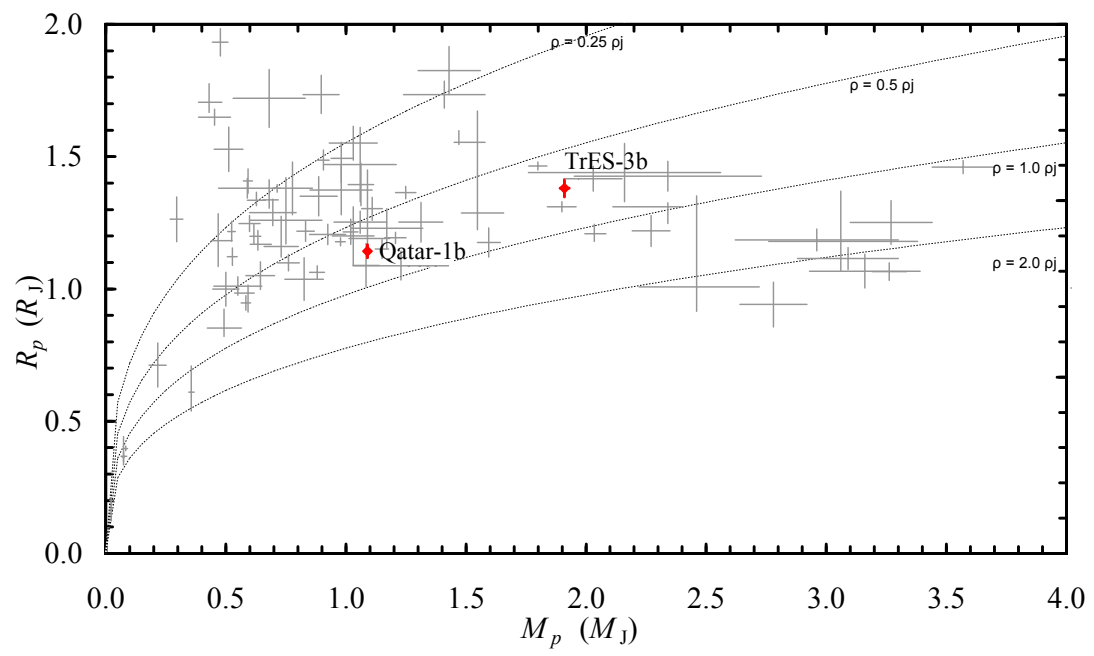

Figure 9: Mass-radius relation of planets. Data apart from measurements of TrES-3b and Qatar-1b (red dots with errors) in this study were obtained from TEP catalogue. Dashed curves, top to bottom, show density, $\rho=0.25,0.5,1.0$ and $2.0 \rho_{J}$ 


\section{Acknowledgements}

This study was supported by TÜBİTAK (Scientific and Technological Research Council of Turkey) under Grant No. 113F353. We thank Çanakkale Onsekiz Mart University Astrophysics Research Centre and Ulupinar Observatory and İstanbul University Observatory Research and Application Center for their support and allowing use of T122 and T60 which were supported partly by National Planning Agency (DPT) of Turkey (project DPT-2007K120660 carried out Çanakkale Onsekiz Mart University) and the Scientific Research Projects Coordination Unit of Istanbul University (project no. 3685). We thank to TÜBİTAK for a partial support in using T100 telescope with project numbers 13CT100523 and 13CT100-537. This paper is part of the Ph.D. thesis of Ç.Püsküllü. This research made use of VIZIER and SIMBAD databases at CDS, Strasbourg, France. We also thank TRESCA data providers and provider's observatories. In this respect, we thank Marc Bretton from Baronnies Provencales Observatory, Chen Cao from Weihai Observatory, Christophe Gillier \& Romain Montaigut from Club Astronomie Lyon Ampere, Erika Pakstiene \& Rimvydas Janulis from VU Institute of Theoretical Physics and Astronomy, Paul Benni from Acton Sky Portal Observatory, Ken Hose from Quarryview Observatory, Juanjo Gonzalez from Cielo Profundo Observatory, Petri Kehusmaa \& Caisey Harlingten from Searchlight Observatory Network, Oleg Mazurenko from Trottier Observatory, Gajdos Stefan \& Ivana Jaksova from Modra Observatory and Marc Deldem from Les Barres Observatory.

Alsubai, K. A., Parley, N. R., Bramich, D. M., West, R. G., Sorensen, P. M., Collier Cameron, A., Latham, D. W., Horne, K., Anderson, D. R., Bakos, G. Á., Brown, D. J. A., Buchhave, L. A., Esquerdo, G. A., Everett, M. E., Fżrész, G., Hartman, J. D., Hellier, C., Miller, G. M., Pollacco, D., Quinn, S. N., Smith, J. C., Stefanik, R. P., Szentgyorgyi, A., Oct. 2011. Qatar-1b: a hot Jupiter orbiting a metal-rich K dwarf star. MNRAS 417, 709-716.

Budding, E., Najim, N. N., Oct. 1980. The system VV ORI and the consistency of photometric analysis of eclipsing binary light curves. Ap\&SS 72, 369-396.

Budding, E., Zeilik, M., Aug. 1987. An analysis of the light curves of short-period RS Canum Venaticorum stars - Starspots and fundamental properties. ApJ 319, 827-835.

Christiansen, J. L., Ballard, S., Charbonneau, D., Deming, D., Holman, M. J., Madhusudhan, N., Seager, S., Wellnitz, D. D., Barry, R. K., Livengood, T. A., Hewagama, T., Hampton, D. L., Lisse, C. M., A’Hearn, M. F., Jan. 2011. System Parameters, Transit Times, and Secondary Eclipse Constraints of the Exoplanet Systems HAT-P-4, TrES-2, TrES-3, and WASP-3 from the NASA EPOXI Mission of Opportunity. ApJ $726,94$.

Ciceri, S., Mancini, L., Southworth, J., Bruni, I., Nikolov, N., D’Ago, G., Schröder, T., Bozza, V., TregloanReed, J., Henning, T., May 2015. Physical properties of the HAT-P-23 and WASP-48 planetary systems from multi-colour photometry. A\&A 577, A54.

Claret, A., Bloemen, S., May 2011. Gravity and limb-darkening coefficients for the Kepler, CoRoT, Spitzer, uvby, UBVRIJHK, and Sloan photometric systems. A\&A 529, A75.

Collins, K. A., Kielkopf, J. F., Stassun, K. G., Dec. 2015. Transit Timing Variation Measurements of WASP-12b and Qatar-1b: No Evidence for Additional Planets. arXiv.

Colón, K. D., Ford, E. B., Lee, B., Mahadevan, S., Blake, C. H., Nov. 2010. Characterizing transiting extrasolar planets with narrow-band photometry and GTC/OSIRIS. MNRAS 408, 1494-1501.

Covino, E., Esposito, M., Barbieri, M., Mancini, L., Nascimbeni, V., Claudi, R., Desidera, S., Gratton, R., Lanza, A. F., Sozzetti, A., Biazzo, K., Affer, L., Gandolfi, D., Munari, U., Pagano, I., Bonomo, A. S., Collier Cameron, A., Hébrard, G., Maggio, A., Messina, S., Micela, G., Molinari, E., Pepe, F., Piotto, 
G., Ribas, I., Santos, N. C., Southworth, J., Shkolnik, E., Triaud, A. H. M. J., Bedin, L., Benatti, S., Boccato, C., Bonavita, M., Borsa, F., Borsato, L., Brown, D., Carolo, E., Ciceri, S., Cosentino, R., Damasso, M., Faedi, F., Martínez Fiorenzano, A. F., Latham, D. W., Lovis, C., Mordasini, C., Nikolov, N., Poretti, E., Rainer, M., Rebolo López, R., Scandariato, G., Silvotti, R., Smareglia, R., Alcalá, J. M., Cunial, A., Di Fabrizio, L., Di Mauro, M. P., Giacobbe, P., Granata, V., Harutyunyan, A., Knapic, C., Lattanzi, M., Leto, G., Lodato, G., Malavolta, L., Marzari, F., Molinaro, M., Nardiello, D., Pedani, M., Prisinzano, L., Turrini, D., Jun. 2013. The GAPS programme with HARPS-N at TNG. I. Observations of the Rossiter-McLaughlin effect and characterisation of the transiting system Qatar-1. A\&A 554, A28.

Eastman, J., Siverd, R., Gaudi, B. S., Aug. 2010. Achieving Better Than 1 Minute Accuracy in the Heliocentric and Barycentric Julian Dates. PASP 122, 935-946.

Fressin, F., Knutson, H. A., Charbonneau, D., O’Donovan, F. T., Burrows, A., Deming, D., Mandushev, G., Spiegel, D., Mar. 2010. The Broadband Infrared Emission Spectrum of the Exoplanet TrES-3. ApJ 711, 374-379.

Gibson, N. P., Pollacco, D., Simpson, E. K., Barros, S., Joshi, Y. C., Todd, I., Keenan, F. P., Skillen, I., Benn, C., Christian, D., Hrudková, M., Steele, I. A., Aug. 2009. A Transit Timing Analysis of Nine Rise Light Curves of the Exoplanet System TrES-3. ApJ 700, 1078-1085.

Hansen, B. M. S., Barman, T., Dec. 2007. Two Classes of Hot Jupiters. ApJ 671, 861-871.

Horne, J. H., Baliunas, S. L., Mar. 1986. A prescription for period analysis of unevenly sampled time series. ApJ 302, 757-763.

Kopal, Z., 1959. Close binary systems. Chapman \& Hall, London.

Kundurthy, P., Becker, A. C., Agol, E., Barnes, R., Williams, B., Feb. 2013. APOSTLE: 11 Transit Observations of TrES-3b. ApJ 764, 8.

Lee, J. W., Youn, J.-H., Kim, S.-L., Lee, C.-U., Koo, J.-R., Feb. 2011. Physical Properties of the Transiting Planetary System TrES-3. PASJ 63, 301.

Lithwick, Y., Xie, J., Wu, Y., Dec. 2012. Extracting Planet Mass and Eccentricity from TTV Data. ApJ $761,122$.

Lomb, N. R., Feb. 1976. Least-squares frequency analysis of unequally spaced data. Ap\&SS 39, 447-462.

Maciejewski, G., Fernández, M., Aceituno, F. J., Ohlert, J., Puchalski, D., Dimitrov, D., Seeliger, M., Kitze, M., Raetz, S., Errmann, R., Gilbert, H., Pannicke, A., Schmidt, J.-G., Neuhäuser, R., May 2015. No variations in transit times for Qatar-1 b. A\&A 577, A109.

Mislis, D., Mancini, L., Tregloan-Reed, J., Ciceri, S., Southworth, J., D’Ago, G., Bruni, I., Baştürk, Ö., Alsubai, K. A., Bachelet, E., Bramich, D. M., Henning, T., Hinse, T. C., Iannella, A. L., Parley, N., Schroeder, T., Apr. 2015. High-precision multiband time series photometry of exoplanets Qatar-1b and TrES-5b. MNRAS 448, 2617-2623.

O’Donovan, F. T., Charbonneau, D., Bakos, G. Á., Mandushev, G., Dunham, E. W., Brown, T. M., Latham, D. W., Torres, G., Sozzetti, A., Kovács, G., Everett, M. E., Baliber, N., Hidas, M. G., Esquerdo, G. A., Rabus, M., Deeg, H. J., Belmonte, J. A., Hillenbrand, L. A., Stefanik, R. P., Jul. 2007. TrES-3: A Nearby, Massive, Transiting Hot Jupiter in a 31 Hour Orbit. ApJ 663, L37-L40.

Petrucci, R., Jofré, E., Melita, M., Gómez, M., Mauas, P., Jan. 2015. Transit timing variation analysis in southern stars: the case of WASP-28. MNRAS 446, 1389-1398.

Poddaný, S., Brát, L., Pejcha, O., Mar. 2010. Exoplanet Transit Database. Reduction and processing of the photometric data of exoplanet transits. NewA 15, 297-301.

Rhodes, M. D., Budding, E., Jun. 2014. Analysis of selected Kepler Mission planetary light curves. Ap\&SS $351,451-471$.

Scargle, J. D., Dec. 1982. Studies in astronomical time series analysis. II - Statistical aspects of spectral analysis of unevenly spaced data. ApJ 263, 835-853.

Southworth, J., May 2008. Homogeneous studies of transiting extrasolar planets - I. Light-curve analyses. MNRAS 386, 1644-1666.

Southworth, J., Nov. 2010. Homogeneous studies of transiting extrasolar planets - III. Additional planets and stellar models. MNRAS 408, 1689-1713.

Southworth, J., Nov. 2011. Homogeneous studies of transiting extrasolar planets - IV. Thirty systems with 
space-based light curves. MNRAS 417, 2166-2196.

Southworth, J., Apr. 2012. Homogeneous Studies of Transiting Planets. In: Richards, M. T., Hubeny, I. (Eds.), From Interacting Binaries to Exoplanets: Essential Modeling Tools. Vol. 282 of IAU Symposium. pp. 131-132.

Sozzetti, A., Torres, G., Charbonneau, D., Winn, J. N., Korzennik, S. G., Holman, M. J., Latham, D. W., Laird, J. B., Fernandez, J., O'Donovan, F. T., Mandushev, G., Dunham, E., Everett, M. E., Esquerdo, G. A., Rabus, M., Belmonte, J. A., Deeg, H. J., Brown, T. N., Hidas, M. G., Baliber, N., Feb. 2009. A New Spectroscopic and Photometric Analysis of the Transiting Planet Systems TrES-3 and TrES-4. ApJ 691, 1145-1158.

Torres, G., Winn, J. N., Holman, M. J., Apr. 2008. Improved Parameters for Extrasolar Transiting Planets. ApJ 677, 1324-1342.

Turner, J. D., Smart, B. M., Hardegree-Ullman, K. K., Carleton, T. M., Walker-LaFollette, A. M., Crawford, B. E., Smith, C.-T. W., McGraw, A. M., Small, L. C., Rocchetto, M., Cunningham, K. I., Towner, A. P. M., Zellem, R., Robertson, A. N., Guvenen, B. C., Schwarz, K. R., Hardegree-Ullman, E. E., Collura, D., Henz, T. N., Lejoly, C., Richardson, L. L., Weinand, M. A., Taylor, J. M., Daugherty, M. J., Wilson, A. A., Austin, C. L., Jan. 2013. Near-UV and optical observations of the transiting exoplanet TrES-3b. MNRAS 428, 678-690.

Vaňko, M., Maciejewski, G., Jakubík, M., Krejčová, T., Budaj, J., Pribulla, T., Ohlert, J., Raetz, S., Parimucha, Š., Bukowiecki, L., Jun. 2013. Photometric follow-up of the transiting planetary system TrES-3: transit timing variation and long-term stability of the system. MNRAS 432, 944-953.

von Essen, C., Schröter, S., Agol, E., Schmitt, J. H. M. M., Jul. 2013. Qatar-1: indications for possible transit timing variations. A\&A 555, A92.

Winn, J. N., Holman, M. J., Torres, G., McCullough, P., Johns-Krull, C., Latham, D. W., Shporer, A., Mazeh, T., Garcia-Melendo, E., Foote, C., Esquerdo, G., Everett, M., Aug. 2008. The Transit Light Curve Project. IX. Evidence for a Smaller Radius of the Exoplanet XO-3b. ApJ 683, 1076-1084. 